Review

\title{
Asthma and COPD: Interchangeable use of inhalers. A document of Italian Society of Allergy, Asthma and Clinical Immmunology (SIAAIC) \& Italian Society of Respiratory Medicine (SIMeR)
}

\author{
Federico Lavorini $^{\text {a, }}{ }^{*}$, Fulvio Braido ${ }^{\text {b }}$, Ilaria Baiardini ${ }^{b}$, Francesco Blasi ${ }^{c}$, \\ Giorgio Walter Canonica ${ }^{\mathrm{b}}$, on behalf of SIAAC-SIMER \\ ${ }^{a}$ Department of Experimental and Clinical Medicine, Careggi University Hospital, Florence, Italy \\ ${ }^{\mathrm{b}}$ Respiratory and Allergy Diseases Clinic, DIMI, University of Genoa, IRCCS AOU San Martino-IST, Genoa, Italy \\ ${ }^{\mathrm{c}}$ Department of Pathophysiology and Transplantation, Università degli Studi di Milano, IRCCS Fondazione Ca' Granda, Ospedale Maggiore Policlinico, Milan, \\ Italy
}

\section{A R T I C L E I N F O}

\section{Article history:}

Received 15 July 2015

Accepted 20 July 2015

Available online 22 July 2015

\section{Keywords:}

Inhalers

Generics

Switching

Asthma

COPD

Adherence

\begin{abstract}
A B S T R A C T
Prescription cost-containment measures are increasing in many European countries and, as more inhaler devices become available, there may be pressure to switch patients from reference inhaled medication to cheaper generic inhaled drugs. Indeed, in some countries, such a substitution is mandated by current regulations, and patients who do not accept the substitution have to pay the difference in cost. Generic inhaled drugs are therapeutically equivalent to original branded options but may differ in their formulation and inhalation device. This new situation raises questions about the potential impact of switching from branded to generic inhaled medications in patients with asthma or chronic obstructive pulmonary disease (COPD), with or without their consent, in countries where this is permitted. Acquisition cost savings from a substitution could be offset by costs related to deterioration in asthma control or worsening in COPD outcomes if the patient is unable or unwilling to use the inhaler device properly. Nonadherence to therapy and incorrect inhaler usage are recognised as major factors in uncontrolled asthma and worsening of COPD outcomes. Switching patients to a different inhaler device may exacerbate these problems, particularly in patients who disagree to switch. Where switching is permitted or mandatory, it is crucial that the reason for switching has been properly explained to the patient and adequate instruction for operating correctly the inhaler have clearly been provided.
\end{abstract}

๑) 2015 Elsevier Ltd. All rights reserved.

\section{Contents}

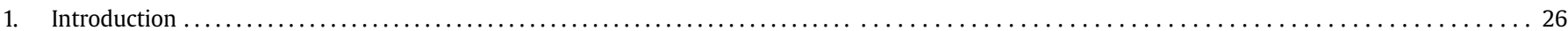

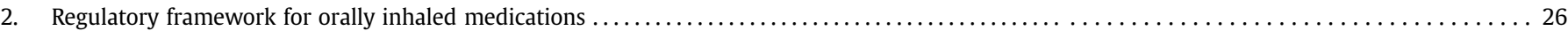

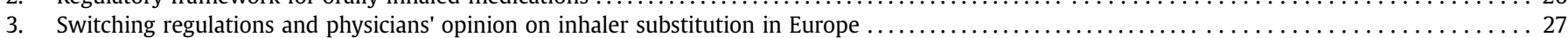

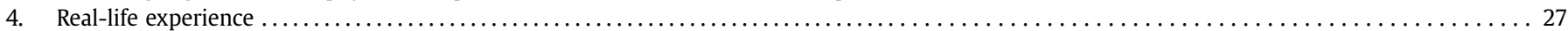

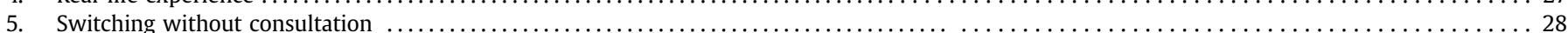

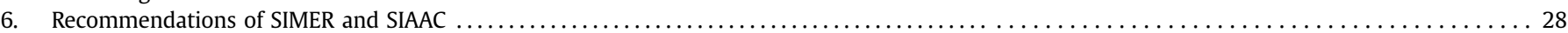

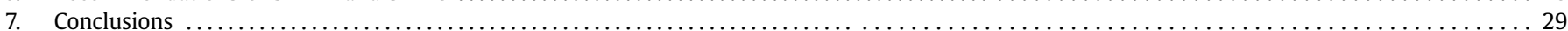

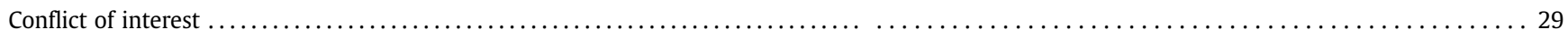

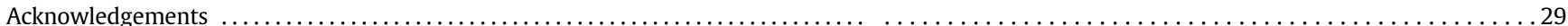

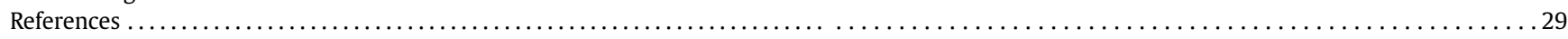

\footnotetext{
* Corresponding author. Department of Experimental and Clinical Medicine, Careggi University Hospital, Largo Brambilla 3, 50134 Florence, Italy.

E-mail address: federico.lavorini@unifi.it (F. Lavorini).
} 


\section{Introduction}

A 3-years observational cohort study showed that persistence with the initial branded treatment in COPD is low, with a substantial proportion of patients changing therapy (switch or add-on therapy). Whether these changes in dispensing patterns are physician-instigated or patient-driven is not clear, neither are fully understood the factors driving the changes but, among them, device acceptability must be considered [1].

Several European countries are experiencing an explosion of new drugs and inhaler devices, either pressurized metered dose inhalers (pMDIs) or dry powder inhalers devices (DPIs), being licensed for patients with asthma or chronic obstructive pulmonary disease (COPD). Some pharma companies have invested much money in research and development of unique new inhaler device platforms for delivering different inhaled drugs. Examples of these new delivery system platforms are the Respimat ${ }^{\circledR}$ soft mist inhaler which is the inhaler of choice for administration of the long-acting antimuscarinic (LAMA) drug Tiotropium and the long-acting beta adrenergic bronchodilator (LABA) drug Olodaterol; the Ellipta ${ }^{\circledR}$ DPI for administration of the LAMA drug Umeclidinium, the LABA drug Vilanterol, and the corticosteroid Fluticasone Furoate; the Genuair ${ }^{\circledR}$ DPI for administration of LAMA drug Aclidinium and LABA drug Formoterol, the Breezhaler ${ }^{\circledR}$ DPI for administration of the LABA drug Indacaterol and the LABA drug Glycopirronium; the Nexthaler ${ }^{\circledR}$ DPI for administration of the fixed combination of the corticosteroid Beclomethasone Dipropionate with the LABA Formoterol; the Spiromax ${ }^{\circledR}$ DPI for the administration of the fixed combination of the LABA drug Formoterol with the corticosteroid Busonide, and the fixed combination of the LABA drug Salmeterol with the corticosteroid Fluticasone Propionate. In addition to these branded inhaled medications, the expiration of the patent protection covering the established inhaled bronchodilators, corticosteroids, and their fixed combinations has contributed to the development of several "generic" inhaled drugs that are bioequivalent [2] to the original reference listed inhaled medications. Generic inhaled medications have the same chemical structure as branded medications but they are not necessarily delivered by the same devices as the original branded options, being the original devices often protected by ongoing patents. Rather generics are delivered by relatively low cost inhalation devices that can vary markedly in design, drug delivery and method of operation than devices of the original branded drugs. The substantial differences that exist in the design of inhaler products makes very difficult to develop generic versions of inhaler products that are interchangeable and substitutable with the originator products. We will face in the next years with newly engineered devices, with the availability of more drugs delivered by the same device but also with the pressure of prescribing cost-containment measures. The last are increasing in many countries and, as more inhalers become available, it is feasible that extending the use of generics is considered an important element to achieve substantial savings theoretically at no detrimental to patient care. Thus, switching patients from reference inhaled drugs to lower-cost "generic" inhaled ones may represent an opportunity for reducing cost of drug treatments in asthma and COPD [3,4].

The present document has been prepared by a working group of Italian Society of Respiratory Medicine (SIMeR) and Italian Society of Allergy, Asthma and Clinical Immunology (SIAACI) with the aim to review the current regulations on generic substitution of marketed reference inhaled medications in Europe. We also question if switching patients from reference to generic inhaled drugs may have potential deleterious effects on asthma control and COPD outcomes. Description of current regulatory requirements associated with the development and submission of dossiers for inhaled medications is outside the scope of this article; excellent descriptions of this aspect can be found in the literature [see e.g. [5-7] also for further references].

\section{Regulatory framework for orally inhaled medications}

When developing a new therapeutically active moiety, the applicant has to provide evidence for the safety, efficacy and quality of the new therapeutically active moiety, as well as the drug product containing the therapeutically active moiety. In case the therapeutically active moiety is known and marketed drug product

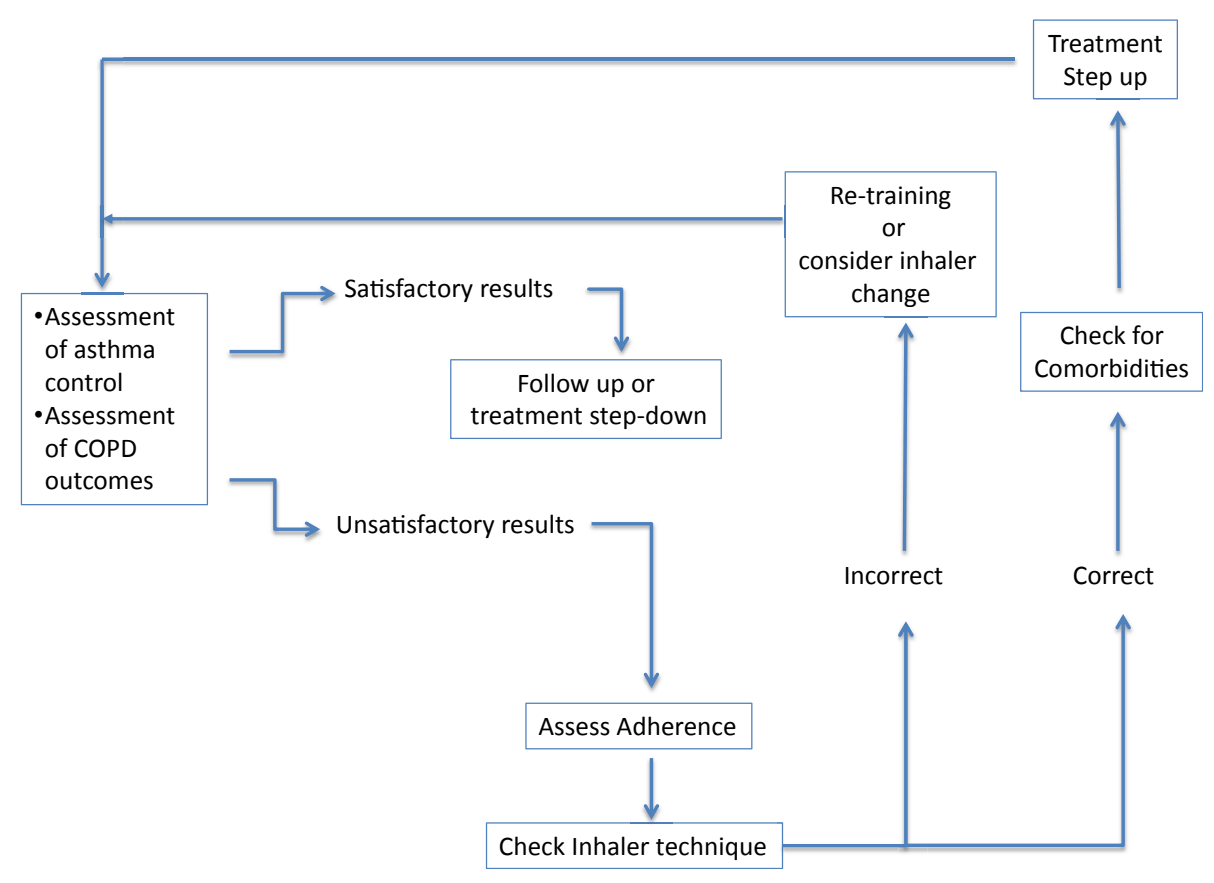

Fig. 1. Treatment algorithm in obstructive lung diseases. 
already exists, a generic applicant will provide evidence on the quality of the new generic product with reference to existing safety and efficacy data when submitting a generic of the product. Although bioequivalence principles have firmly been defined [5], at present there is no international consensus on many of the details regarding the requirements for the design, conduct and evaluation of bioequivalence studies. Consequently, each regulatory authority [see e. g. 8-10] has issued its own corresponding guidelines.

According to the European directive [11], a generic product must contain the same active substance in the same amount as the reference product and demonstrate its therapeutic equivalence, which is defined as sufficiently comparable efficacy and safety profile of the test and reference products so that a clinically relevant difference between products can be reliably excluded [8]. It is responsibility of regulatory authorities to ensure that a generic product is equivalent to the originator product in all important respects, and to assure patients and prescribers that there is no significant difference between the two products in the clinic [11]. The European Medical Agency (EMA) guideline provides the possibility to consider approving a generic version of an inhaler product based on in vitro assessment in case the product meets all the stringent criteria set forth in the guideline that leads to the assumption that the generic version of the product is therapeutic interchangeable [8]. In case the in vitro comparability is not given, lung deposition tests are required that might have to be complemented by pharmacodynamics and eventually clinical studies. Even so demonstrating equivalent bioavailability at the site(s) of action for orally inhaled products has been proposed as a suitable way to demonstrate essential similarity, the EMA made clear that "orally inhaled products are definitively not "generics' but hybrids" and that "simple bridging to the bioequivalence model is mostly not sufficient." [8].

In the US, orally inhaled drug products are considered combinations products and are defined by the Food and Drug Administration (FDA) as "therapeutic and diagnostic products that combine drugs, devices, and/or biological products", in which the device, the formulation and the patient interact in a complex manner to deliver a specific dose of fine drug particles to the different regions in the lung $[9,10]$. Moreover, each device differs from the other making the development of a generic product that is therapeutically interchangeable very challenging [10]. The FDA requires generic manufacturers to demonstrate equivalent delivery to the lungs, equivalent systemic exposure with the generic product and comparability of the pMDI or the DPI they propose to use to that of the branded product [10]. However, there is no consensus on the protocols for the necessary studies [12]. In addition, the excipients in the generic product must be qualitatively and quantitatively the same as those in the reference product. Due to the difficulties in demonstrating bioequivalence between generic and reference inhaled drugs, FDA received few applications for generics, even though many of the older pMDI products are on the market without patent or exclusivity protection. The FDA recognises the challenges that these requirements present and is currently working with manufacturers, regulatory bodies and academia to find solutions to them $[6,12]$.

\section{Switching regulations and physicians' opinion on inhaler substitution in Europe}

In Europe, regulations on generic substitution of marketed drugs vary from country to country [4]. In some countries (i. e. Germany and Finland), substitution of branded inhaler medications is mandated by current regulations, while in others (i. e. the UK and the Netherlands) substitution is permitted if generic names are used on the prescription. To ensure continuity of use with a particular device, the phrase 'medical necessity' must be used on the prescription. Interestingly, the Lung Alliance Netherlands has been working with healthcare insurers to develop guidelines that specifically address the conditions under which switching to the insurer's preferred (lower cost) inhaler device might be acceptable; at the present, more than 80 inhalers currently available in the Netherlands can be considered interchangeable [13]. In Italy, generic inhalers are available and pharmacists may switch respiratory patients from one combination of drug and inhaler device to a generic unless the prescription specifies that the patient must not be switched. Patients who do not accept the substitution of a branded product with a generic have to pay the difference in cost. In Spain regulatory and expert bodies advocate for the safety of patients and their ability to have a voice in treatment decisions, especially in the case of patients who are established on a longterm treatment regime. At variance with the above mentioned countries, in France there are no generics for inhaled products that can be substituted for branded products, although some pMDIs could be considered interchangeable.

A survey of healthcare professionals in the UK [14] found that the vast majority of them were concerned about potential problems arising from prescriptions that do not specify the inhaler to be dispensed, and $46 \%$ of the interviewed physicians were aware of actual incidents in which patients received unfamiliar inhaler including patient confusion, ineffective inhaler technique and the need to reissue prescriptions. In another survey [15] conducted at the primary and secondary care level in Germany, the Netherlands and UK one third of the physicians interviewed considered the inhaler before considering the chemical entity within a class of treatments and over half the respondents reported problems with the inhaler as one of the main reasons for switching inhaled therapy. A Delphi process was undertaken with four leaders in respiratory medicine to identify important themes relating to interchangeability of DPIs [15]. These themes were developed into a structured questionnaire to use with the respondents in France, Germany and UK. Only 9\% of the physician interviewed thought that DPIs were interchangeable while the remaining considered these inhalers not interchangeable [15]. In addition, over $90 \%$ of the physicians thought that interchangeability of DPIs would have a negative impact on patients adherence and inhaler handling and on willingness to use the inhaler if the patients was not involved in the choice of the device. Furthermore, about $80 \%$ of physicians thought that substitution of a patient's regular DPI with another could have a negative impact on asthma control [15]. The majority of physicians were opposed to substitution of one DPI for another device if the pharmacist does not consult the patient and/or the physician. Taken together, the results of these surveys indicated that healthcare professionals perceive inhaler devices as different and not interchangeable, with physicians opposed to substitution of one device by another without consultation with the patient. In addition, healthcare professionals believe patients involvement in the inhaler choice to be essential for adherence to therapy.

\section{Real-life experience}

The pMDIs and DPIs are the most frequently prescribed inhaler devices in Europe [16]; they differ in their efficiency of drug delivery to the lower respiratory tract, depending on the design of the device, its internal resistance, the formulation of the medication, its particle size, the velocity of the produced aerosol plume, and how easily the patients can use the device. The efficiency of drug delivery may also be influenced by patients' preference, which, in turn, affects patients' adherence to treatment and, indeed, the subsequent long-term control of the disease. DPIs in particular can vary markedly in design and method of operation [17] and this could lead to different handling errors in a real-life context [18]. When long-term users of branded inhaled drugs are dispensed 
generics delivered by a different inhaler they are likely to be unfamiliar with the new device and could become aware of a change in taste/sensation [19]. This may reduce their confidence in the efficacy of the generic drug, increasing the risk of poor compliance and possibly loss of asthma control [20]. There was an example of this in 2005, when New Zealand's medication funding authority removed the subsidy on the branded salbutamol pMDI $\left(\right.$ Ventolin $\left.{ }^{\circledR}\right)$, intending to gradually replace it with the cheaper generic, the Salamol ${ }^{\mathbb{R}}$ pMDI [21]. Bioequivalence between Ventolin and Salamol pMDIs has been demonstrated in patients with asthma [21]. However, since its introduction to New Zealand anecdotal reports [22] of decreased therapeutic effect, inhaler blockage and unpleasant taste were reported. Reti et al. [23] found that half of those patients converted to the Salamol pMDI withdrew prematurely due to ineffectiveness and that, in those who continued to use Salamol, nearly all had worse asthma stability. In contrast, Chang et al. [24] found no difference in the efficacy of Salamol and Ventolin pMDIs in relieving bronchoconstriction. Differences in the patient population, study design and methodology used may account for the conflicting results. However, it should be noted that Chang et al. [24] administered both salbutamol formulations using pMDIs in conjunction with spacers, thus affecting drug deposition and consequently the effectiveness of both preparations. An earlier study showed that failure to shake the canister before each inhalation halved the systemic availability of budesonide from a pMDI compared with that from the pMDI used with a spacer [25], confirming that suboptimal usage can have a profound effect on drug delivery from a pMDI and that use of a spacer can mitigate this variability [25].

\section{Switching without consultation}

Successful management of asthma and COPD is dependent on a number of factors including correct inhaler use [26]. Current asthma [27] and COPD [28] guidelines emphasise the importance of training patients how to use their inhalers correctly, and report that medications are not interchangeable on a 'mcg' or 'per puff basis because newer delivery devices may deliver an higher therapeutic dose to the lungs. All guidelines and recommendations state that, whatever device is selected, patients should be instructed in its use and undergo regular checking of inhaler technique [29,30]. Patients are more likely to achieve better asthma control and COPD outcomes as a result of successful dose delivery when they become familiar with a particular device. However, guidelines provide no guidance on how inhaler technique training would be provided when a patient is switched from a branded drug to a generic alternative, particularly if disagree with the switching ("non-consent switching"). Non-consented switch is defined as the substitution of a delivery device (and medication) without prior approval or knowledge by the patient. This means that patients do not receive any counselling from their healthcare provider about the new medication and device, which may result in poor inhalation technique and lead to loss of disease control [31]. Concerns regarding this phenomenon were identified in the US, where former New York City Public Advocate, Mark Green, has been highly critical of Pharmacy Benefit Managers in Health Management Organisations switching patients' medication for purely financial reasons [32]. Moreover, Lipton et al. [33] also reported how Pharmacy Benefit Managers exert their influence to change prescribing decisions on cost grounds. Thomas et al. [31] retrospectively evaluated the impact on asthma control of steroid inhaler switching without an accompanying visit or consultation in the UK. Of note, over half of device switches were from DPIs to pMDIs. Compared with matched controls, patients whose steroid inhaler was switched were significantly more likely to experience unsuccessful asthma treatment [31]. These results were confirmed more recently by Doyle et al. [19] who showed that switching asthma patients' inhalers without their consent may diminish the self-control associated with good asthma management, leave the doctor-patient relationship damaged, increase resource utilisation, and waste medication. It is therefore possible that non-consent switch may not result in cost savings, because of increases in clinic visits for education and support and negative impacts on asthma control, resulting in higher short and long-term healthcare costs. Patient education and involvement in treatment decisions can improve adherence to therapy [17] but adherence is likely to decline in patients who have treatment switched without consultation [31,32]. Non-compliance rates already range from 16 to 50\% among patients with asthma and COPD and this contributes to the morbidity, mortality and associated costs of these conditions $[31,32]$. Increasing the risk of poor adherence by switching inhalers without consultation is likely to add to these problems. In the case of switching, it is crucial that the reason for the switch has been properly explained to the patient and instructions for operating the device correctly have been clearly demonstrated; a routinely monitoring of inhaler technique at each visit is also mandatory.

\section{Recommendations of SIMER and SIAAC}

- Over the next few years it is expected that a number of new branded inhaled drugs as well as the generics of expired patented medicines will become available.

- The increasing range of inhaler devices represents an opportunity for physicians and pharmacists to optimise inhalation therapy and technique.

- Switching inhaler devices is recommended only as part of a concordant asthma or COPD review where inhaler technique is assessed and patient agreement with the switching is obtained.

- Prescription of generic inhaled drugs is rightly being encouraged to reduce expenditure, particularly at the primary care level, nevertheless appropriate patient's instruction about the use of the inhaled drugs must be guaranteed.

- Switching patients from one inhaled drugs to another delivered by a different device may have a negative impact on patient adherence to the treatment and consequently disease control.

- A patient, who is familiar and stabilized on one type of inhaler, should not be switched to another device without his/her involvement and follow-up education.

- Where switching is considered, adequate patient's instruction on correct inhaler use must be guaranteed.

- Pharmacists are in an excellent position to educate patients about inhaler technique because they are the last healthcare professionals seen by patients before an inhaled medication is used. However, they should guarantee that patients are dispensed the same inhaler device type for each prescription.

- Simplification of inhalation therapy by prescription of one instead of multiple inhaler device types must be encouraged.

- Due to the typical time-course of asthma and COPD, switching from different doses, drugs and, often, inhalers might happen in daily clinical practice. Therefore, particular attention has to be deserved to this critical issue.

A practical algorithm on the assessment of asthma control and COPD outcomes is depicted in Fig. 1. If the patient is unable to use a particular inhaler correctly despite repeated attempts, a change in inhaler device should be considered. In the cases where ongoing, uncontrolled asthma or worsening of COPD outcomes persists in the face of a correct inhaler technique, then therapy should be stepped up according to the treatment guidelines and another appointment scheduled in order to recheck the symptoms. 


\section{Conclusions}

In a national survey conducted on a group of about 2000 individuals forming a representative sample of the italian population, iinhaled drugs were recognised as the most common treatment for asthma and COPD by 65\% of the respondents[34]. Most of the respondents tended to attribute positive characteristics to the devices with regard to safety, reliability, effectiveness, ease of use and practicality. Approximately $75 \%$ of the respondents indicated that an inhaler device is the best solution for respiratory diseases and $45 \%$ believed that inhaler devices could also be useful for other diseases. Approximately nine patients out of 10 stated that their physician provided an adequate explanation regarding the inhalation technique [34]. However, a high proportion of patients do not have the competence to use their device effectively because they have forgotten what they were taught and no longer apply the correct technique that they were trained to use [34]. For this reason, international guidelines for asthma and COPD management state that inhalation technique should be assessed regularly and corrected if it is inadequate [34].

For the above mentioned reasons caution should be taken when switching patients from one device to another one. This is particularly true for DPIs, which differ widely in the way they operate, their appearance and delivery characteristics [17]. Indeed, patient preference for different DPIs varies [17], indicating that they are not all the same from the patient's perspective, although patient preference for a device does not ensure either improved compliance or adherence. Thus, inhaler devices should be prescribed with no switching of device without the involvement of both physician and patient [35]. Should substitution of a generic for a branded inhaler be permitted, safeguards are required to ensure that patients receive adequate training and are willing to use the new device. Most relevant, the responsibility for such training needs to be clarified because physicians may not be aware that a prescribed device has been replaced with a generic drug at the pharmacy [36]. When evaluating the potential cost benefits of switching inhalers, all relevant costs should be considered, including those arising from additional consultations, the time required for training and the management of any subsequent acute events. These costs, plus the likelihood of repeat consultations and prescriptions among patients switched to cheaper, less familiar devices, may outweigh any cost benefits obtained from switching.

\section{Conflict of interest}

FL has received in the last three years speaker honoraria from AstraZeneca, Boehringer Ingelheim, Chiesi, Cipla, GlaxoSmithKline, TEVA.

FB has received in the last three years speaker or consultant honoraria or research funding from: A. Menarini - Almirall AstraZeneca - Boeringher Ingelheim - Chiesi Farmaceutici - Glaxo Smith Kline - Lab. Guidotti - Malesci - Mundifarma - Novartis Teva - Valeas - Zambon.

IB has no conflict of interest.

GWC has received in the last three years speaker or consultant honoraria or research funding from: A. Menarini - Almirall AstraZeneca - Boeringher Ingelheim - Chiesi Farmaceutici - Glaxo Smith Kline - Lab. Guidotti - Malesci - Mundifarma - Novartis Teva - Valeas.

Fulvio Braido has received in the last three years speaker or consultant honoraria from: A. Menarini - Almirall - AstraZeneca Boeringher Ingelheim - Chiesi Farmaceutici - Glaxo Smith Kline Lab. Guidotti - Malesci - Mundifarma - Novartis - Zambon Dompè - Almirall.

\section{Acknowledgements}

Authors thank the Italian Society of Allergy, Asthma and Clinical Immmunology (SIAAIC) and the Italian Society of Respiratory Medicine (SIMeR) for the scientific support in developing this paper.

\section{References}

[1] F. Penning-van Beest, M. van Herk-Sukel, R. Gale, J.W. Lammers, R. Herings, Three-year dispensing patterns with long-acting inhaled drugs in COPD: a database analysis, Respir. Med. 105 (2011 Feb) 259-265.

[2] P. Daley-Yates, D. Parkins, Establishing bioequivalence for inhaled drugs: weighing the evidence, Exp. Opin. Drug Deliv. 8 (2011) 1297-1308.

[3] D. Price, M. Summers, P. Zanen, Could interchangeable use of dry powder inhalers affect patients? Int. J. Clin. Pract. 59 (Suppl. 149) (2005) 3-6.

[4] F. Lavorini, V. Ninane, J. Haughney, L. Bjermer, M. Molimard, R.P. Dekhuijzen, Switching from branded to generic inhaled medications: potential impact on asthma and COPD, Expert Opin. Drug Deliv. 10 (2013) 1597-1602.

[5] A. García-Arieta, J. Gordon, Bioequivalence requirements in the European Union: critical discussion, AAPS 14 (2012) 738-748.

[6] C. Evans, D. Cipolla, T. Chesworth, et al., Equivalence considerations for orally inhaled products for local action-ISAM/IPAC-RS European Workshop report, J. Aerosol Med. Pulm. Drug Deliv. 25 (2012) 117-139.

[7] A. Fuglsang, The US and EU regulatory landscapes for locally acting generic/ hybrid inhalation products intended for treatment of asthma and COPD, J. Aerosol Med. Pulm. Drug Deliv. 25 (2012) 243-247.

[8] European Medicines Agency Committee for Medicinal Products for Human Use (CHMP), in: (CHMP) CfMPfHU (Ed.), Guideline on the Requirements for Clinical Documentation for Orally Inhaled Products (OIP) Including the Requirements for Demonstration of Therapeutic Equivalence between Two Inhaled Products for Use in the Treatment of Asthma and Chronic Obstructive Pulmonary Disease, 2009. London.

[9] US Food and Drug Administration US FDA, Guidance for the In Vitro Portion of Bioequivalence Requirements for Metaproterenol Sulfate and Albuterol Inhalation Aerosol, 1989.

[10] US Food and Drug Administration US FDA, Drug-development Approval Process. Scientific and Regulatory Considerations for Bioequivalence (BE) of Dry Powder Inhalers (DPIs), 2011.

[11] http://www.edctp.org/fileadmin/documents/ethics/Directive_200183EC of the european parliament.pdf. (accessed: 1.05.2013).

[12] W.P. Adams, R.C. Ahrens, M.L. Chen, et al., Demonstrating bioequivalence of locally acting orally inhaled drug products (OIPs): workshop summary report, J. Aerosol Med. Pulm. Drug Deliv. 23 (2010) 1-29.

[13] Lung Alliance Network, Correct Use of Inhaled Medication Asthma and COPD, 2011. Amersfoort.

[14] A. Buchanan, H. Pinnock, J. Barnes, Generic prescribing of breath actuated and dry powder inhalers in the UK, Prim. Care Respir. J. 11 (2002) 95.

[15] D. Price, Do healthcare professionals think that dry powder inhalers can be used interchangeably? Int. J. Clin. Pract. Suppl. 149 (2005) 26-29.

[16] F. Lavorini, C.J. Corrigan, P.J. Barnes, et al., Retail sales of inhalation devices in European countries: so much for a global policy, Respir. Med. 98 (2011), 1099-1013.

[17] W. Azouz, H. Chrystyn, Clarifying the dilemmas about inhalation techniques for dry powder inhalers: integrating science with clinical practice, Prim. Care Respir. J. 15 (2012) 208-213.

[18] M. Molimard, C. Raherison, S. Lignot, et al., Assessment of handling of inhaler devices in real life: an observational study in 3811 patients in primary care, J. Aerosol Med. 16 (2003) 249-254.

[19] S. Doyle, A. Lloyd, A. Williams, H. Chrystyn, et al., What happens to patients who have their asthma device switched without their consent? Prim. Care Respir. J. 19 (2010) 131-139.

[20] PHARMAC, New Zealand Pharmaceutical Schedule Update Effective 1 July 2005, PHARMAC, Wellington, 2005.

[21] D. Dockhorn, J. Vanden Burgt, et al., Clinical equivalence of a novel nonchlorofluorocarbon-containing salbutamol sulfate metered-dose inhaler and a conventional chlorofluorocarbon inhaler in patients with asthma, J. Allergy Clin. Immunol. 96 (1995) 50-56.

[22] J. Gillies, J. Brown, C. Byrnes, et al., PHARMAC and ventolin in New Zealand, N. Z. Med. 120 (2005) 1220.

[23] S. Reti, Ventolin to Salamol - a crossover study in New Zealand, N. Z. Med. 119 (2006) $1-7$.

[24] C. Chang, M. Cooray, G. Mills, R. Hancox, Is Salamol less effective than Ventolin? A randomized, blinded, crossover, study in New Zealand, N. Z. Med. 120 (2007) 1267

[25] L. Thorsson, S. Edsbäcker, Lung deposition of budesonide from a pressurized metered-dose inhaler attached to a spacer, Eur. Respir. J. 12 (1998) 1340-1345.

[26] F. Lavorini, M. Levy, P. Dekhuijzen, G. Crompton, Inhaler choice and inhalation technique: key factors for asthma control, Prim. Care Respir. J. 18 (2009) 241-242.

[27] GINA Report, Global strategy for asthma management and prevention, 
December 2011 update. Available from: http://www.ginasthma.org/.

[28] GOLD. Global Strategy for Diagnosis, Management, and Prevention of COPD (2011 Update): Global Initiative for Chronic Obstructive Lung Disease, 2011. Available from: http://www.goldcopd.org/.

[29] http://www.worldallergy.org/UserFiles/file/

GWCManifestoAdherenceChicago_fullpage.pdf.

[30] F. Braido, I. Baiardini, F. Blasi, R. Pawankar, G.W. Canonica, Adherence to asthma treatments: 'we know, we intend, we advocate', Curr. Opin. Allergy Clin. Immunol. 15 (2015 Feb) 49-55.

[31] M. Thomas, D. Price, H. Chrystyn, et al., Inhale corticosteroids for asthma: impact of practice level device switching on asthma control, BMC Pulm. Med. 9 (2009) 1 .

[32] M. Green, Compromising Your Drug of Choice: How HMOs are Dictating Your
Next Prescription, Public Advocates Office, New York City, 1996. www pubadvocate.nyc.gov.

[33] H.L. Lipton, D.H. Kreling, T. Collins, K.C. Hertz, Pharmacy benefit management companies: dimensions of performance, Annu. Rev. Public Health 20 (1999) $361-401$.

[34] F. Braido, I. Baiardini, M. Sumberesi, F. Blasi, G.W. Canonica, Obstructive lung diseases and inhaler treatment: results from a national public pragmatic survey, Respir. Res. 14 (2013 Sep 22) 94.

[35] R. Booker, Do patients think that dry powder inhalers can be used interchangeably? Int. J. Clin. Pract. 59 (Suppl. 149) (2005) 30-32.

[36] A. Williams, H. Chrystyn, Survey of pharmacists' attitude towards interchangeable use of dry powder inhaler, Pharm. World Sci. 29 (2007) 221-227. 